\title{
CAMBIOS EN LAS ESTRUCTURAS DE LOS HOGARES RURALES \\ Formas de adaptación y resiliencia
}

\author{
CHANGES IN RURAL HOUSEHOLD STRUCTURES \\ Forms of adaptation and resilience
}

Luis Camarero Rioja Icamarero@poli.uned.es

Universidad Nacional de Educación a Distancia (UNED). España.

Julio A. del Pino Artacho jadelpino@poli.uned.es

Universidad Nacional de Educación a Distancia (UNED). España

\begin{abstract}
ResUmen
Este artículo trata sobre la capacidad de las estructuras de hogar para responder a las condiciones ambientales. En primer lugar, se expone la relación entre sostenibilidad demográfica y resiliencia de los hogares en las áreas rurales. A continuación, se presentan los mecanismos básicos de la adaptación entre las estructuras de hogar y entorno socioeconómico a través de las teorías de Chayánov y Le Play. Se analiza empíricamente la España rural, donde se constata una etapa de modernización de las estructuras de hogar, seguida más recientemente de cierta diversificación. Dentro de este proceso, destaca el número de hijos que alargan el periodo de convivencia con sus padres, constituyendo estrategias similares a las tradicionalmente empleadas por las familias troncales. En el actual contexto de las áreas rurales, las estructuras de hogar muestran su condición resiliente ante los cambios socioeconómicos.
\end{abstract}

\section{Palabras Clave}

Áreas rurales; España; Estructura de hogar; Resiliencia social.

\section{Abstract}

This paper examines the capacity of household structures to respond to environmental conditions. First, it looks at the relationship between demographic sustainability and the resilience of rural households. Afterwards, it examines basic mechanisms of adaptation between household structures and the socioeconomic environment based on the theories of Chayanov and Le Play. The empirical research, carried out in rural Spain, found that household structures first underwent a process of modernization, followed more recently by certain diversification. Within this process, what stands out is the number of adult children that extend the period of cohabitation with their parents, constituting similar strategies to those traditionally employed by stem families. In the current context of rural areas, household structures have shown resilience in the face of socioeconomic changes.

\section{KEYWORDS}

Household Structure; Rural areas; Social Resilience; Spain. 


\section{SOSTENIBILIDAD DEMOGRÁFICA Y HOGARES RESILIENTES*}

Dentro del contexto generalizado de envejecimiento creciente en el que se encuentran las sociedades europeas, las áreas rurales de España muestran, comparativamente con las áreas urbanas, un sobreenvejecimiento motivado fundamentalmente por los efectos del intenso proceso de éxodo rural de la segunda mitad del siglo XX. En el medio rural, al importante crecimiento en la esperanza de vida y de reducción de la fecundidad, que caracterizan la segunda transición demográfica (Van de Kaa 1987), se añade el vaciamiento por emigración de ciertas generaciones y el impacto que dicha emigración tiene sobre las sucesivas. El resultado es una relación de mayores sobre generaciones jóvenes mucho más elevada que lo que los procesos meramente vegetativos producen. Además, el continuado éxodo feminizado juvenil, introduce otra diferencia significativa de la población rural respecto a la de las áreas urbanas: la población rural se encuentra masculinizada (Camarero et al. 2009) En este contexto deben considerarse las formas y las diferencias de convivencia familiar y doméstica entre áreas rurales y urbanas. En las áreas rurales hay una dificultad mayor para la formación de nuevos núcleos y un mayor peso de hogares con ancianos solos o conviviendo con otros familiares.

Así pues, una inspección de las estructuras de población constata las dificultades de cara al futuro de las poblaciones rurales. Dicho de otra forma, demográficamente las poblaciones rurales son poco sostenibles.

¿Qué quiere decir la expresión "poco sostenibles"? Se refiere fundamentalmente a las dificultades de reproducción demográfica, dificultades que inciden en el desenvolvimiento económico y cultural (Brown 2008; Brown y Schafft 2011). Precisamente, la definición original y más genérica de sostenibilidad hace referencia a la relación entre generaciones ${ }^{1}$. Es evidente que la interacción entre generaciones es función de la relación en términos numéricos entre las mismas. Por ejemplo, el sistema europeo de protección social se ha basado en que las generaciones activas, siempre más numerosas, se hacían cargo de las generaciones pasivas, siempre menos numerosas. Las estructuras tan desequilibradas de las poblaciones rurales tienen consecuencias en múltiples aspectos relativos al desarrollo económico, como son la organización de los mercados de trabajo y de la vida económica -actividades que no pueden desarrollarse por falta de mano de obra. Pero de forma quizás más dramática en la propia representación identitaria de la comunidad —-por ejemplo, cuando no se pueden completar equipos

"El presente trabajo se inscribe dentro del proyecto del programa de I+D+i CSO2008-01286 (Movilidad espacial, mercados de trabajo y sostenibilidad social) y se ha beneficiado de la Ayuda Complementaria CSO2011-15703

1"Development that meets the needs of the present without compromising the ability of future generations to meet their own needs" (WCED 1987: 326). 
deportivos-y en la reorganización de la tradicional división y asignación de actividades comunitarias - por ejemplo, la organización de las fiestas.

Que la población rural tenga amenazada su sostenibilidad no quiere decir ni mucho menos que desaparezca. Los sistemas sociales también son resilientes. Es decir no están absolutamente determinados por el entorno sino que poseen la capacidad de actuar y responder a los cambios del entorno. Las poblaciones rurales hacen frente a su situación de desequilibrio y difícil sostenibilidad aumentando la resiliencia (McManus et al. 2012; Trabalzi y de Rosa 2012).

En su origen, el concepto de resiliencia procede de los sistemas mecánicos y se refería a la capacidad que tienen los objetos de absorber las modificaciones que produce el entorno. Desde la ecología se ha venido definiendo como la capacidad adaptativa del sistema (Holling 2001). Una forma de hibridación del concepto es la "resiliencia evolutiva": "el término resiliencia se refiere tanto a la capacidad de las poblaciones para permanecer en el estado actual (análogo al de resistencia en la literatura ecológica) como a la de llevar a cabo adaptaciones evolutivas en respuesta a los cambios en las condiciones" (Sgro et al. 2011: 327).

Holling y Gunderson (Gunderson y Holling 2001; Holling 2001) se preocupan de abordar la capacidad de anticipación de los sistemas sociales respecto a las variaciones del entorno. Sus trabajos inspiran distintos análisis empíricos, especialmente en comunidades aisladas y muy dependientes de los recursos naturales del entorno ${ }^{2}$. Especialmente Adger (Adger 2000; Adger et al. 2002) ha estudiado las acciones de respuesta de las comunidades ante cambios ambientales, encontrando que la acción resiliente de las comunidades era pequeña.

No obstante, el concepto de resiliencia no puede llevarse, desde los sistemas ecológicos, mediante una mera analogía, a los sistemas sociales. Estos, a diferencia de los sistemas físicos y ecológicos, se componen por sistemas de interacción (Luhmann 1995). $O$ como ha señalado Giddens (1979), se forman mediante la indisoluble relación entre 'agencia' y 'estructura'. Es precisamente ahí, en la interdependencia entre agente y estructura donde se regula la respuesta - la resiliencia - frente a las modificaciones del entorno. En esta línea resulta central el trabajo de Bennett (2004) sobre la resiliencia de las estructuras patriarcales en áreas rurales.

Desde la tesis de la estructuración, Pelling y Manuel-Navarrete (2011) analizan la resiliencia respecto al cambio medioambiental en dos centros urbanos de México, destacando la importancia que tiene el sistema de interacción para sostener la inercia y, por tanto, la rigidez sobre la flexibilidad. El sistema de interacción reduce la resiliencia y frena las acciones tendentes a la sostenibilidad ambiental. Esta línea de trabajo apunta hacia el carácter poco elástico de los sistemas sociales.

\footnotetext{
${ }^{2}$ La mayor parte de ellos publicados en la revista Ecology and Society, con el impulso de The Resilience Alliance.
} 
Otros trabajos empíricos sobre comunidades diferenciadas étnicamente en áreas desarrolladas, como en Nueva Zelanda (Scott et al. 2000), han puesto el acento en la incorporación de cuestiones como la equidad y la participación. Consideran que las diferencias étnicas, de clase social, de género y de ocupación obstaculizan la sostenibilidad. El debate acerca de la idea de sostenibilidad ha tenido un importante impacto en la teoría de la justicia. Agyeman (2005) ha propuesto el término de 'just sustainability' para mostrar que el debate sobre la sostenibilidad en el fondo es un debate sobre derechos, tanto entre coetáneos como con relación a futuras generaciones. Hermans y Knippenberg (2006) han desarrollado el concepto de resiliencia como "justicia generacional". Para estos autores mientras la justicia se refiere a la relación intrageneracional, la resiliencia lo hace a las relaciones intergeneracionales.

Desde la noción de justicia — universal y atemporal— la sostenibilidad transforma una demanda social en un elemento central de la agenda política (Agyemany Evans 2004). La sostenibilidad es un problema de carácter social. Y como problema social pertenece en primer lugar a las comunidades. Como han señalado Marsden y Hines (2008: 27): "Both, community and sustainability, need each other".

McManus et al. (2012) se han acercado a la resiliencia en las comunidades rurales preguntándose por qué, a pesar del continuo declive -demográfico, económico y social-, no desaparecen las poblaciones que representan el 'deep rural'. Para estos autores la respuesta vuelve a incidir en el sistema de interacción. Podría deducirse que si el sistema de interacción reduce la resiliencia, las comunidades rurales en las áreas de fuerte declive económico, demográfico y social, se mantendrían por propia inercia, sin saber cómo afrontar el futuro. Lo que estos autores sugieren, sin embargo, es una estrategia de mantenimiento como respuesta a la situación de declive. Frente a la resiliencia evolutiva de Holling, que exige sistemas dinámicos para que respondan al medio ambiente, McManus y su equipo recurren a la idea de "adaptación estable" y refieren la resiliencia como un mecanismo para preservar la estructura: "La capacidad de un sistema para absorber las perturbaciones y mantener su estructura y función básica" (Walker y Salt 2006: 1). En sus hallazgos encuentran que el "sentimiento de pertenencia" resulta central para superar el fuerte aislamiento. El mecanismo de resiliencia pasa por el reforzamiento de las relaciones primarias y por la defensa del capital social y del estatus adquiridos con anterioridad.

Los resultados de los distintos trabajos empíricos vienen a mostrar que hay dos orientaciones fundamentales para observar la resiliencia social: la externa y la interna. La externa, cuando únicamente se analiza la respuesta del conjunto del sistema social en relación con el medio ambiente; se supone que la sociedad tiende a ajustarse a los cambios ambientales y desde esta postura se enfatiza la falta de flexibilidad del sistema de interacción. La interna, cuando se busca la respuesta a los cambios ambientales dentro del propio sistema social, destacando el mantenimiento de las estructuras.

Este segundo enfoque es el que tiene interés en nuestro propósito de exploración de los procesos sociales de absorción de los desequilibrios. Si bien el sistema de interacción defiende a la estructura, también esta debe adaptarse para defender al sistema 
de interacción. En este sentido, lo que queremos analizar aquí son las transformaciones de los grupos domésticos, estructura clave de la reproducción —además de lugar de convivencia intergeneracional—como pieza esencial de la sostenbilidad social.

Los hogares conforman sistemas de interacción fundamentales, basados en las relaciones íntimas, conformando elementos clave de la estructura social.

Los desequilibrios generacionales y la merma en la capacidad reproductiva tiene lógicamente su efecto en los hogares, estos tendrán que adaptarse y a la vez hacer frente a dicha situación. Es decir, son una unidad de resiliencia. Son afectados por el entorno, lo que les hace desarrollar mecanismos de adaptación y, especialmente, de resolución de los desequilibrios.

El hogar es, en gran medida, la realización tangible de las estructuras familiares. El carácter de las relaciones familiares es largo en el tiempo y el hogar funcionalmente adquiere el carácter de diferenciar el grupo doméstico del entorno.

El hogar es una estructura resiliente, que trata de defender a sus miembros de las variaciones del entorno. Por ejemplo, el nivel de empleo o de servicios sociales hace que los hogares se compongan de una o de otra forma. Es decir, para defender a sus miembros, el hogar absorbe las alteraciones del entorno modificando la composición del grupo familiar. La regulación de la fecundidad, la edad al matrimonio o los sistemas de herencia son, entre muchos otros, algunos de los principales mecanismos reguladores de la composición de los hogares.

\section{ADAPTACIÓN Y CAMBIO DE LOS HOGARES EN EL ENTORNO RURAL}

Una vez señalado el carácter resiliente de las estructuras de convivencia, nos interesa examinar los mecanismos que posibilitan esta capacidad de adaptación. En el ámbito rural, los hogares se configuran como entidades que conectan las estructuras demográficas con las estructuras socioeconómicas. Por una parte, los hogares se construyen a partir de determinadas distribuciones de población —que asimismo contribuyen a edificar, mediante el emparejamiento y la filiación, o su ausencia. Por otra parte, los hogares se organizan para dar respuesta a los problemas de producción y reproducción social en el nivel micro, siendo sus actores fundamentales.

En relación con las particularidades de la familia rural, el clásico estudio de Chayánov (1925/1966) examina la comunidad rural alrededor de un sistema socioproductivo de carácter familiar, relativamente autónomo. De este modo, la organización económica rural basada en las unidades familiares mostraba el funcionamiento de un modo de producción campesino diferenciado sobre la base, no de la explotación capitalista de clase, sino de la autoexplotación de la familia campesina basada en la satisfacción de necesidades. Tal modo de producción descansa en la familia como sujeto del sistema, unidad de producción y consumo. Para Chayánov la familia campesina obedece a un equilibrio entre las necesidades de consumo y la fuerza de trabajo familiar para satis- 
facerlas. La familia ajusta su tamaño y composición a esta función socioeconómica, de modo que actúa como fuerza de trabajo fundamental de la unidad económica. De este modo, habrá que tener en cuenta no solo el número de miembros sino su capacidad de producción y la relación entre los miembros productores y los meramente consumidores. Resulta evidente que, en gran medida, esta relación depende de la estructura demográfica por edad y sexo (especialmente cuando se habla de ciertos trabajos en determinados contextos). Asimismo, Chayánov es consciente de la importancia de la dinámica familiar, de los cambios de los grupos domésticos a lo largo del tiempo. De este modo, habrá que tener en cuenta tanto las transformaciones internas de los hogares derivadas del paso del tiempo (el ciclo del hogar que va situando progresivamente al hogar típico en una situación de mayor capacidad laboral, una vez que los hijos dependientes comienzan a integrar la fuerza laboral de la unidad familiar) como los cambios del hogar original derivados de la salida o entrada de nuevos miembros, formando nuevos núcleos o haciéndolos desaparecer. Tal sería el caso de las nuevas familias creadas por los hijos del núcleo original, o la integración en el núcleo original de un progenitor anciano.

La asociación entre formas familiares y capacidad de explotación económica actúa a lo largo del tiempo, mediante la existencia de diversos modelos de tenencia y transmisión de la propiedad, que representan pautas de ajuste estructural entre explotaciones y familias. Este aspecto, al que Chayánov no presta una atención específica, resulta el segundo elemento que conectaría, junto a la capacidad de generar rentas mediante el trabajo, la composición de los hogares con el ámbito socioeconómico.

La relación entre las formas familiares y la transmisión patrimonial es una cuestión que preocupó en la primera sociología de la familia. Frédéric Le Play (1884) analiza la familia teniendo en cuenta el sistema de herencia, distinguiendo las familias patriarcales y las troncales. En las familias patriarcales (famille patriarcale, joint families, familias extensas) se van sumando generaciones al grupo familiar que contribuyen, a la producción en la explotación y el mantenimiento de la unidad doméstica, sin división del patrimonio, bajo el férreo control del cabeza de familia. Solo cuando el tamaño familiar resulta inviable para el mantenimiento de la explotación surgen divisiones. Por otro lado, las familias troncales (famille souche, stem families) presentan un mecanismo institucional de regulación para el adecuado mantenimiento del tamaño de las explotaciones a través de la herencia. En este caso, solo uno de los hijos varones casados convive con los padres, en tanto que los hermanos permanecen en el hogar únicamente estando solteros, y optan por emigrar o formar hogares ajenos en el momento de emparejarse. La transmisión patrimonial se compromete en el momento del casamiento y articula una madeja de relaciones entre los padres, el hijo casado y la nuera, que incluye el aseguramiento del cuidado por parte de los padres (Verdon 1979).

Le Play distinguió, junto a la familia patriarcal y la familia troncal, un tipo de familia, correspondiente a las sociedades urbanas e industrializadas, que calificaría de "inestable" y que se aproxima a lo que se conoce como familia nuclear. Sería una familia formada por los padres y los hijos, cuyo ciclo vital incluye la neolocalidad de las parejas de los hijos y se disuelve a la muerte de los padres, dividiéndose el patrimonio entre los 
hijos. En la estela de Le Play, pero ajena a su reformismo moralizante, buena parte de la sociología asumió la idea de que la modernización incluía en el ámbito familiar el paso desde formas extensas de familia hacia formas nucleares (Parsons 1949). Y, en términos territoriales, las formas extensas irían relacionadas con el ámbito rural (aunque también allí se encontraría en decadencia debido a la orientación mercantil de la actividad agraria), mientras que las formas nucleares corresponderían al ámbito urbano ${ }^{3}$.

En las sociedades industrializadas de mediados del siglo XX, el paso hacia la familia nuclear moderna redefinía el papel económico de la familia mediante la salarización de los individuos, en el terreno productivo, y la reorganización de la función reproductiva de la familia una vez inscrita en el conjunto más amplio de las políticas de bienestar. La familia moderna queda así constituida como unidad de consumo y agente socializador, dejando atrás su papel de unidad de producción y consumo.

El individualismo señalado por Le Play representa la misma fuerza que más de un siglo después dará una nueva vuelta de tuerca a la institución para llevarla desde la familia nuclear hacia la "familia postfamiliar" (Beck y Beck-Gersheim 2001). El proceso de individualización socava los lazos consanguíneos y la perdurabilidad de las alianzas para componer un nuevo escenario donde el grupo doméstico tiende a fundarse sobre afinidades electivas y su supervivencia depende cada vez más de la acción de los individuos en contextos estructuralmente restringidos. Como consecuencia, las estructuras familiares tienden a presentar contornos algo más diversos y flexibles, aparecen menos sólidas y determinadas por modelos normativos. No obstante, los hogares siguen representando estrategias posibles de supervivencia doméstica, formas de convivencia que permiten la sostenibilidad del hogar y la realización de los individuos.

En este contexto, tal y como estudiaran Chayánov y Le Play subrayando distintos aspectos, las formas de convivencia actúan como estructuras que tratan de adaptarse a las circunstancias socioeconómicas y demográficas de la población. Siguiendo con la tipología de Le Play, la familia troncal se configura como un recurso organizativo que, tomado de un modo flexible (como mayorazgo o minorazgo, con cabeza de familia en la generación mayor o en la intermedia, etc.), permite una gran adaptabilidad a diversos escenarios. Por el contrario, la familia patriarcal resulta un recurso muy poco flexible en circunstancias de cierta individualización. Finalmente, la familia nuclear podría presentar problemas en la gestión del ámbito reproductivo por la ausencia de apoyos, especialmente en la situación de transformación en las relaciones de género y de desequilibrios demográficos propia de las áreas rurales.

\footnotetext{
${ }^{3}$ Esta concepción se ha puesto en tela de juicio desde la demografía histórica a partir de los trabajos de Laslett (1965) y Hajnal (1965), en los que se afirma la particularidad de las poblaciones históricas de Europa occidental y Norteamérica, donde la familia nuclear ya venía siendo común al igual que el retraso de la nupcialidad. Tales supuestos solo invalidarían parcialmente la hipótesis sociológica de la modernización familiar, referida exclusivamente al tránsito desde las sociedades tradicionales a las sociedades industriales en los siglos XIX y XX.
} 


\section{ANÁLISIS DE LAS ESTRUCTURAS DE HOGAR RURALES}

La indagación que motiva este artículo consiste en determinar la capacidad que las estructuras tienen para responder a las condiciones ambientales. Se trata de estudiar las formas familiares como estructuras resilientes en relación con un medio ambiente, caracterizado particularmente en el ámbito rural por la escasez de efectivos y, sobre todo, por el desequilibrio generacional y de género.

En este sentido, se han presentado dos mecanismos tradicionales que han actuado en el medio rural para configurar los hogares en relación con la supervivencia económica: el ajuste entre la capacidad de explotación del trabajo familiar y el tamaño de los hogares, y los sistemas de propiedad y herencia de las explotaciones, como reguladores que optimizan la viabilidad de los hogares. Según este esquema, la composición de los hogares respondería al cumplimiento de sus funciones y a su propia sostenibilidad.

Con la modernización, la imbricación del escenario rural dentro de una estructura socioeconómica ampliada, centrada en el ámbito urbano industrial, supuso importantes cambios para las estructuras de hogar rurales. En el ámbito socioeconómico, las áreas rurales proveían insumos laborales para la nueva economía industrial, a la vez que se desmantelaba el sistema tradicional de captación de rentas mediante la explotación de la fuerza de trabajo familiar y de reproducción patrimonial. Como consecuencia de ello, las formas de hogar articuladas en torno al viejo modelo socioeconómico, como la familia patrimonial o la troncal, perdieron importancia frente al modelo de hogar nuclear, mucho más adecuado a la función socializadora y de consumo hacia la que se orientaban los hogares.

Sin embargo, el hecho de que el contexto socioeconómico de las áreas rurales haya cambiado no significa que los hogares hayan perdido esa capacidad de adaptación. En este sentido, los hogares han dejado de orientarse hacia el ámbito productivo, que ha pasado a ser dominado por estructuras económicas independientes de la economía doméstica —como la cooperativa, la industria agroalimentaria, la empresa turística o la administración-, y tienden a configurarse alrededor del ámbito reproductivo, como gestores fundamentales de cuestiones como la dependencia, el trabajo doméstico, las redes de ayuda o el capital social; vínculos que sostienen el entramado social local, haciendo posible que comunidades aparentemente insostenibles en términos demográficos o económicos permanezcan.

Nuestras pesquisas se encaminan, pues, primero, a constatar el efecto general de la modernización sobre las estructuras de hogar rural; especialmente en términos de reducción del tamaño del hogar y nuclearización, lo que sin duda concuerda con el proceso de convergencia entre las áreas rurales y urbanas como producto de su inserción en una misma estructura socioeconómica. Para ello, utilizaremos los datos censales sobre "tipos de hogar", comparando 1970 - primer censo con datos sobre la estructura de los hogares- y el último censo disponible, el de 2001. Se analiza tanto el tamaño promedio del hogar como su estructura, lo que nos permite enjuiciar las dos tendencias 
modernizadoras más relevantes de las formas de hogar: la reducción del tamaño y la nuclearización.

En relación con los indicadores de estructura de hogar se ha utilizado la única tipología empleada en 1970, que nos permite la comparación de hogares en relación con la ausencia o presencia de uno o más núcleos familiares y la existencia o no de personas añadidas al núcleo, emparentadas o no. A pesar de los cambios en los conceptos de hogar, familia y núcleo entre 1970 y 2001, la comparación es ajustada, refiriéndonos en todo caso a la convivencia de una o más personas bajo el mismo techo (hogar) ${ }^{4}$ y a las relaciones familiares principales -emparejamiento y filiación-(núcleo) ${ }^{5}$.

En segundo lugar, dada la incidencia de los desequilibrios demográficos sobre la estructura de hogares, se pretende explorar las formas de convivencia de la población rural -no de los hogares rurales - controlando el efecto de la estructura por edad y analizando el problema de la transición generacional, es decir, estudiando el momento en el que una generación toma el relevo de la anterior como referencia del hogar. Dada la hegemonía de la familia nuclear y, por lo tanto, de la convivencia de dos generaciones en el hogar, el estudio de la emancipación y la transición generacional permite explorar los mecanismos que rigen el cumplimiento de las funciones reproductivas del hogar rural contemporáneo, distinguiendo que los hogares se encuentren comandados por la generación de los padres — como venía siendo habitual en la sociedad rural tradicional—o por la de los hijos - como se fue imponiendo en las ciudades. En este sentido, es posible que sociedades con un acusado desequilibrio demográfico den lugar a estructuras de hogar particulares que permitan el sostenimiento de una población sobreenvejecida y masculinizada como la rural.

La fuente utilizada a estos efectos es la Encuesta de Presupuestos Familiares (EPF) de 2009, que analiza una muestra nacional de 22.346 hogares, de los que 5.673 son hogares rurales, situados en municipios con menos de 10.000 habitantes, garantizando la significatividad de las estimaciones, una vez establecidos los factores de ponderación

\footnotetext{
${ }^{4}$ En 1970, a efectos censales, la familia se define como "el grupo de personas vinculadas generalmente por parentesco que hacen vida en común, ocupando normalmente la totalidad de la vivienda". Desde 1991, el concepto censal de familia es sustituido por el concepto de hogar que, en 2001, viene definido simplemente como "el grupo de personas residentes en la misma vivienda familiar". En todo caso se excluyen aquellas personas que viven en establecimientos colectivos (y no, por lo tanto, en viviendas familiares) o, en términos de 1970 , que no viven en familia.

${ }^{5}$ El núcleo familiar se refiere a los principales vínculos familiares, en términos de emparejamiento y filiación. En el Censo de 1970, donde no aparece definido expresamente, se aludía simplemente a la constitución de cuatro tipos de núcleos familiares: matrimonio sin hijos, matrimonio con hijos, padre con hijos y madre con hijos.

Desde 1991, se detalla que el núcleo familiar restringe la noción de familia al contemplar exclusivamente los vínculos más estrechos, es decir, los antedichos, con la excepción de contemplar la cohabitación, es decir, las parejas no casadas; y añadiendo la especificación de que los hijos deben estar solteros y no emparejados. Establece asimismo algunas reglas de delimitación de núcleos (preferencia de la relación padre/madre-hijo/a sobre la relación hijo/a-padre/madre), cuyo efecto en la comparación no puede evaluarse.
} 
correspondientes a la afijación estratificada por comunidades autónomas de la encuesta. Además, la EPF tiene la virtud de recoger información muy precisa sobre la estructura de los hogares. La elección del año 2009 responde a la capacidad de comparación con investigaciones anteriores y, particularmente con la Encuesta a la Población Rural (Camarero et al. 2009).

Los indicadores analizados corresponden, en primer lugar, a la proporción de población en la que conviven al menos dos generaciones emparentadas (por vínculo filial directo - hijos- o electivo - parejas de los hijos-), según la edad en grupos quinquenales. Asimismo, para establecer la transición generacional, se utiliza la proporción de población en posición de sustentador principal del hogar o pareja, dentro de los hogares donde conviven dos generaciones emparentadas. Finalmente se profundiza en las estructuras de hogar de la generación-soporte 6 (30-49 años), según la convivencia con otras generaciones, y se analiza la relación entre envejecimiento y lo que se ha dado en llamar "neomayorazgo", como forma familiar particular en la que un hijo soltero (especialmente los varones) convive con sus padres.

En cuanto al indicador de ruralidad utilizado, debido a los datos publicados en 1970, se recurre en la primera comparación de hogares al tamaño de las entidades de población o "tipo de zona", incluyendo bajo la rúbrica de lo rural no solo la zona rural (hasta 2.000 habitantes) sino también la intermedia (2.001-10.000 habitantes). Sin embargo, los indicadores, extraídos de la EPF, utilizan el tamaño de población (municipios con menos de 10.001 habitantes). Dado el objetivo de nuestro trabajo y la independencia de los aspectos investigados a través de cada fuente, la diferencia de indicadores de ruralidad no afecta a la validez de la investigación.

\section{EL PROCESO DE MODERNIZACIÓN Y DIVERSIFICACIÓN DE LOS HOGARES RURALES}

En España, el estudio de las estructuras familiares en relación con el territorio se ha centrado en la explicación de los cambios mediante el marco teórico de la modernización. Así, encontramos cierta evidencia acerca de la modernización de las estructuras de hogar rurales (Gómez-Reino 1967; Sancho Hazak 1972; De Pablo 1970; Del Campo 1982; Del Campo y Navarro 1985), en términos de nuclearización y reducción del tamaño de los hogares. Por otra parte, existe una preocupación por la insostenibilidad de las estructuras reproductivas rurales, como consecuencia de desequilibrios demográficos arrastrados y los constantes procesos de abandono selectivo -como el de las mujeres con estudios - (Camarero et al. 2009). En vez del hogar, aparecen otros sujetos sociales, como los mayores, los jóvenes, las mujeres o los hombres, individualizados,

\footnotetext{
${ }^{6}$ La generación soporte supone un grupo generacional clave en las áreas rurales para el sostenimiento de poblaciones envejecidas (Camarero et al. 2009).
} 
trazando trayectorias laborales y familiares, de movilidad y de logro (Díaz Méndez 1997; González, De Lucas y Ortí 1985; Camarero 2005). Estas trayectorias resultaban relativamente ajenas al devenir del grupo primario del que formaban parte, en el entendido de que la familia se comportaba como una estructura coercitiva y fijadora, más bien ajena al mundo productivo mercantilizado que representaba la modernización.

Debido a la rapidez de los cambios familiares en España, se ha sostenido que el proceso de modernización caracterizado por la hegemonía de la familia nuclear se ha visto superpuesto por una segunda ola de cambios familiares, que redundan, fundamentalmente, en la pluralidad de formas familiares (Iglesias y Flaquer 1993; Jurado 2008). Tales cambios se sostienen sobre dos procesos demográficos básicos: el envejecimiento de la población y la caída de la fecundidad, que definen la "segunda transición demográfica" (Van de Kaa 1987).

Lamentablemente, se carece de datos censales sobre estructuras familiares anteriores a 1970. Por ello, nos centraremos en verificar, por una parte, la persistencia del proceso de modernización, que en 1970 ya se encontraba avanzado y, por otra, en contrastar las tendencias de la segunda oleada de cambios, especialmente en relación con la realidad rural.

La modernización de las estructuras familiares en España es clara, tanto si atendemos al número de miembros como al predominio de la familia nuclear. En promedio, el número de miembros se ha reducido en más de un miembro en treinta años y no ha dejado de disminuir tampoco durante el último decenio, como observamos en la tabla 1. Las diferencias en este aspecto entre las zonas urbanas y rurales son muy pequeñas en términos globales, presentando incluso las áreas rurales un tamaño ligeramente inferior. Sin duda, ello es debido al envejecimiento de la población rural y la concentración en edades altas de hogares de una o dos personas. Así que si reducimos la comparación a la generación soporte, entre 30 y 49 años, entonces encontramos que los hogares rurales albergan de media más miembros que los urbanos, de acuerdo al cierto retraso supuesto en la reducción del número de miembros por hogar.

Tabla 1.

Variación en el tamaño medio de los hogares rurales y urbanos.

\begin{tabular}{lcc}
\hline & Rural & Urbano \\
\hline 1970 & 3,92 & 3,91 \\
2001 & 2,85 & 2,87 \\
2009: Total & 2,66 & 2,68 \\
2009: Generación soporte & 3,25 & 3,10 \\
\hline
\end{tabular}

Nota: Para 1970 y 2001 las áreas rurales se refieren a la variable censal 'Tipo de zona: rural e intermedia'; en 2009 se utiliza un tamaño municipal inferior a 10.000 habitantes.

Fuente: Instituto Nacional de Estadística (Censo de Población 1970 y 2001 y Encuesta de Presupuestos Familiares 2009). 
En segundo lugar, encontramos un predominio claro de las familias nucleares en todo el periodo (tabla 2). Dos de cada tres hogares está formado por familias nucleares en sentido estricto (sin ninguna persona ajena al núcleo en el hogar), habiéndose incrementado la proporción durante el periodo analizado. Aunque los datos no son estrictamente comparables, todo apunta a que la nuclearización no ha hecho sino incrementarse durante el último decenio, superando ya el $70 \%$ de los hogares. La nuclearización parecía esencialmente completada en 1970, tanto para el entorno rural como para el urbano. De hecho, las estructuras familiares rurales presentaban un rasgo de nuclearización exclusiva más fuerte que las urbanas (los núcleos con otras personas, emparentadas o no, se reducían al $13 \%$ frente al $16 \%$ urbano). En 2001, la nuclearización exclusiva se ha reducido significativamente en áreas rurales (del 69 $\%$ al $62 \%$; las urbanas del $69 \%$ al $66 \%$ ), pero la ampliación del núcleo ha pasado a ser más importante en las áreas rurales que en las urbanas. Es decir, la nuclearización se ha visto acompañada en las áreas rurales de una diversidad de formas familiares mayor que en las ciudades.

El proceso de nuclearización viene acompañado de una reducción en las formas extensas de hogar. Los hogares de dos o más núcleos han visto reducido su peso a la mitad durante el periodo estudiado. Estos grupos se encuentran tradicionalmente más

Tabla 2.

Estructura del hogar rural y urbano, 1970 y 2001.

\begin{tabular}{|c|c|c|c|c|c|}
\hline & & \multicolumn{2}{|c|}{1970} & \multicolumn{2}{|c|}{2001} \\
\hline & & Urbana & Rural & Urbana & Rural \\
\hline \multirow{3}{*}{ Sin núcleo } & Unipersonal & 6,5 & 8,6 & 19,6 & 21,6 \\
\hline & Personas emparentadas & 2,8 & 2,3 & 3,0 & 3,2 \\
\hline & Personas no emparentadas & 0,9 & 0,3 & 1,1 & 0,7 \\
\hline \multirow{3}{*}{1 núcleo } & Sin otras personas & 68,6 & 68,9 & 66,0 & 61,6 \\
\hline & $\begin{array}{l}\text { Con otras personas (alguna del servicio } \\
\text { doméstico/no emparentadas) }\end{array}$ & 1,9 & 0,3 & 1,6 & 1,2 \\
\hline & $\begin{array}{l}\text { Con otras personas (ninguna del servicio } \\
\text { doméstico/emparentados) }\end{array}$ & 14,4 & 12,7 & 6,3 & 8,0 \\
\hline \multirow{2}{*}{2 núcleos } & Padres e hijos/emparentados & 4,4 & 6,2 & 2,0 & 3,3 \\
\hline & Otros & 0,4 & 0,4 & 0,3 & 0,2 \\
\hline 3 núcleos o más & Familias con 3 núcleos o más & 0,1 & 0,3 & 0,1 & 0,2 \\
\hline TOTAL & & $100 \%$ & $100 \%$ & $100 \%$ & $100 \%$ \\
\hline
\end{tabular}

Fuente: Censo de Población 1970 y 2001, Instituto Nacional de Estadística. 
asociados a los hogares rurales, y en ellos se encuentran, definidas de modo estricto, las familias troncales y patriarcales.

Así pues, aunque con modesta relevancia numérica, se encuentra que en los entornos rurales se ha producido un proceso de nuclearización matizado tanto por una cierta complejidad de los núcleos (con aumento de personas vinculadas ajenas al núcleo) como por la importancia relativa que han continuado teniendo las familias polinucleares. Estos indicios podrían avalar la hipótesis, ya apuntada por Solsona y Treviño (1990), de que dentro del proceso de modernización familiar, marcado por la nuclearización, caben ciertas variaciones que tienden a aumentar la complejidad del hogar nuclear (con vinculación entre dos núcleos o con otras personas ajenas). Estas variaciones pueden entenderse dentro de las relaciones intergeneracionales en un contexto demográfico envejecido, en el que las cargas del trabajo reproductivo se sobrellevan a través de organizaciones domésticas de tamaño y estructura óptima, como se observa más adelante.

Finalmente, otro de los rasgos más sobresalientes de los cambios en las estructuras familiares tiene que ver con su diversificación. Los procesos de transición residencial y de formación de hogares en las grandes cohortes nacidas durante el baby boom se han visto retardados y diversificados con el consiguiente descenso en la formación de nuevos hogares (Camarero et al. 2009). Junto al patrón generalizado, que obedece a un retraso en el calendario del emparejamiento y del alumbramiento del primer hijo, existe una cierta diversificación de las formas de hogar, con repuntes aún pequeños pero significativos de los hogares multipersonales que no forman familia. En la tabla 2 se observa que los hogares sin núcleo formados por dos o más personas han crecido notablemente, especialmente en los entornos rurales y fundamentalmente debido a los hogares de personas emparentadas que no forman núcleo (por ejemplo, hermanos o primos).

En conclusión, la evolución de la estructura de hogares de la España rural refleja que se partía ya en 1970 de una situación de claro predominio de las familias nucleares que, no obstante, se ha intensificado. Estrechamente vinculada a la evolución demográfica, se ha producido una explosión de hogares unipersonales. Y, aunque de un modo más débil, se percibe cierta diversificación de las formas de los hogares rurales, proceso que se superpone a la reducción de formas familiares extensas, ligadas teóricamente a etapas anteriores a la modernización. Tales características confirman, en general, tanto las hipótesis relacionadas con la modernización (hegemonía del núcleo único y disminución del tamaño de los hogares), como la de la pluralización de las formas familiares.

\section{LA CONVIVENCIA INTERGENERACIONAL}

Parece evidente que dentro del panorama de fuerte desequilibrio generacional los hogares rurales tienen que verse afectados de forma significativa. En este sentido deberían observarse modificaciones en la convivencia intergeneracional, es decir, en cuanto a la presencia de una o más generaciones en el mismo hogar. 
Ruggles y Heggeness (2008) señalan que la convivencia intergeneracional debería guardar relación con el desarrollo económico, en el sentido -expresado por Le Play (1884) y Goody (1963) - de reducción del número de generaciones que conviven en el mismo hogar por la progresiva prevalencia de la familia nuclear. Sin embargo, en el estudio que realizan estos autores, se constata, con los datos de la evolución durante el último cuarto de siglo pasado, por una parte, que no existe la reducción esperada en la convivencia intergeneracional; y por otra, algo que resulta aún más sorprendente: que en los hogares de convivencia intergeneracional, por regla general, las generaciones mayores siguen manteniendo la responsabilidad como "cabezas de familia".

Esta última observación resulta de un extraordinario interés analítico. La convivencia intergeneracional como estrategia de organización familiar resulta muy distinta en función de qué generación se sitúa como dependiente. En las sociedades agrarias, la generación dependiente era la de los hijos, que, aun teniendo su propia familia, residía bajo la autoridad económica paterna, a la espera de la sucesión. En las sociedades urbanas contemporáneas esta tendencia cambia. En la medida en que los ingresos no son del conjunto familiar, se constituye una familia nuclear a la que se añaden los ascendientes en una posición secundaria dentro de la organización doméstica, máxime si dicha convivencia es motivada por la necesidad de los mayores de recibir cuidados.

Como han señalado Gómez, González y Hazak (1999), además de una reducción del peso de las familias agrarias, en el medio rural también se ha venido produciendo una progresiva desfamiliarización de las actividades relacionadas con la agricultura. Según estas consideraciones, en el caso de las áreas rurales deberíamos esperar una reducción de la convivencia intergeneracional en la medida en que la familia pierde su carácter unitario como organización productiva y las generaciones no dependen de una actividad familiar compartida. Por ello es esperable que, al igual que en las áreas urbanas, prime la familia nuclear.

En líneas generales, la progresiva reducción de la actividad agraria como actividad familiar a la par que la creciente diversificación de actividades en las áreas rurales permitiría una mayor posibilidad de independencia económica y, por tanto, también residencial, de forma que se constataría, además de la reducción del tamaño del hogar, un cambio de funcionalidad de la convivencia. Los casos de convivencia estarían ahora menos relacionados con la organización de la subsistencia y más relacionados con el apoyo mutuo entre las distintas generaciones. Es decir, en cierta medida la dependencia generacional se transforma en algunos casos en solidaridad intergeneracional. Pilcher (1995) ha destacado cinco funciones de la convivencia, una vez que los hijos alcanzan el estatus de adultos: apoyo económico - especialmente de padres a hijos-, apoyo en el alojamiento — generalmente de padres a hijos —, cuidados personales — de hijos a

\footnotetext{
${ }^{7}$ Ruggles (2010) señala que en las familias europeas existe una "aversión" a la convivencia de los padres o suegros en el hogar de los hijos. Pilcher (1995) confirma que en el caso inglés solo el $6 \%$ de los mayores de 65 años reside con sus hijos.
} 
padres - , ayudas prácticas — también de hijos a padres - y emocionales y morales — compartida entre las distintas generaciones. Es decir, la convivencia respondería a una estrategia consciente de apoyo mutuo entre distintas generaciones

Los datos señalan que la convivencia generacional sigue siendo importante en las áreas rurales españolas. Los gráficos que registran la convivencia entre padres e hijos para las distintas cohortes permiten, a través de la técnica de la cohorte ficticia ${ }^{8}$, reconstruir el patrón de salida del hogar paterno (gráfico 1). En el caso de España, la emancipación se hace a edades relativamente tardías. Aproximadamente a la edad de 27 años la mitad de los jóvenes viven aún con sus padres. Pero la principal cuestión no es el retraso en la emancipación residencial de las áreas rurales, sino la elevada prevalencia que tiene la convivencia intergeneracional en edades tardías. En las áreas rurales españolas uno de cada seis habitantes de entre 40 y 60 años sigue viviendo con sus padres, cifra que en las áreas urbanas es del $10 \%$.

Es decir la población rural española mantiene una convivencia intergeneracional más larga a la vez que también, para un porcentaje importante, esta convivencia se convierte en permanente en el tiempo. Un efecto importante es el hecho de que se observa que el cuidado de mayores incide en un repunte de la convivencia intergeneracional de las mujeres rurales de 50 a 60 años. (Ver gráfico 2).

\section{Gráfico 1.}

Personas que residen con sus padres o suegros según edad.

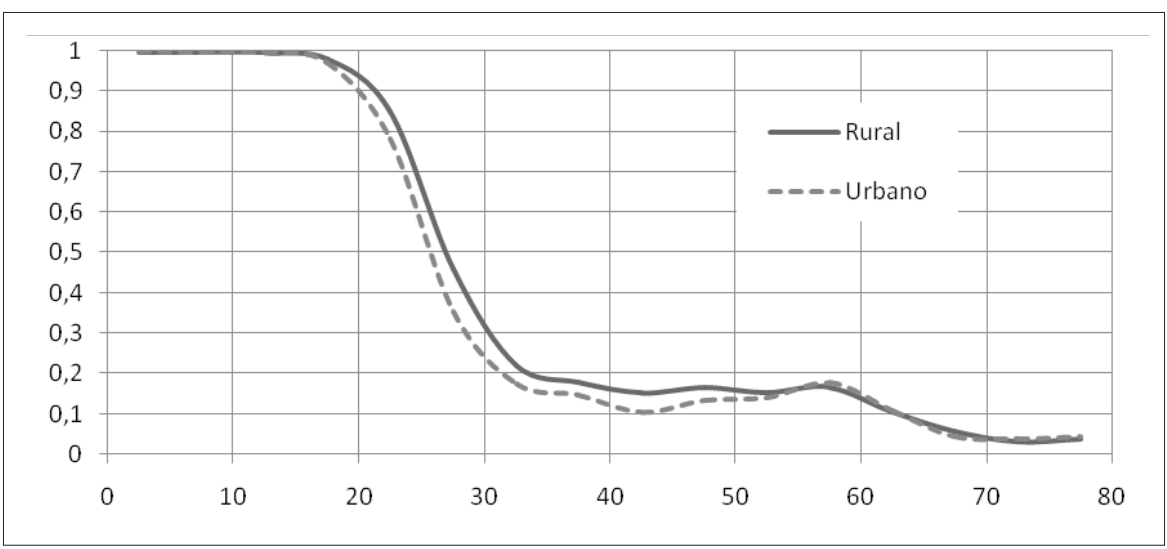

Fuente: (España) Encuesta de Presupuestos Familiares 2009, Instituto Nacional de Estadística.

\footnotetext{
${ }^{8}$ Aplicación de las tasas actuales de convivencia intergeneracional a una generación hipotética, de modo que pueda describirse la trayectoria típica de este indicador a lo largo del ciclo vital de las personas. En este caso, la generación se mide mediante las marcas de clase de la edad en años cumplidos agrupada en intervalos de diez.
} 
Gráfico 2.

Hombres y mujeres que residen con sus padres o suegros en las áreas rurales según edad.

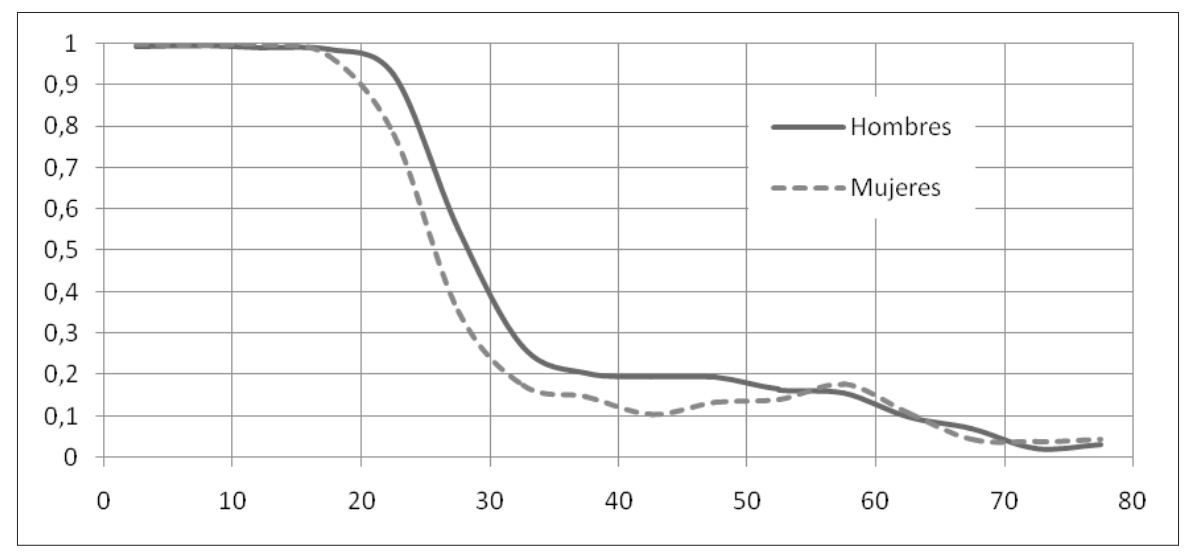

Fuente: (España) Encuesta de Presupuestos Familiares 2009, Instituto Nacional de Estadística.

El hecho de que en España se mantenga la convivencia generacional en edades tardías dirige la investigación a una nueva relación de preguntas: la de cuál es la generación de las dos que actúa como cabeza de hogar, la de los padres o la de los hijos, y la de cuándo se produce el cambio entre generaciones respecto a la responsabilidad en la dirección del hogar. Proceso este que podemos denominar como transición generacional. Si bien es difícil valorar cuándo es el momento en que se produce, podemos utilizar como indicador para comparar distintas situaciones la edad en que los sustentadores principales $^{9} 0$ sus parejas superan a los que son sustentados (ver gráfico 3). En los gráficos se ha calculado la mediana de la distribución de forma gráfica.

Los datos señalan que la transición generacional se hace en edades elevadas. En el caso de España y aproximadamente con 47 años, la mitad de quienes conviven generacionalmente están en la posición de sustentador principal o su pareja, como padres, mientras que la otra mitad siguen en la posición de hijos. Al respecto no hay diferencias entre hogares rurales y urbanos. Definitivamente, los datos señalan que el retraso en la

${ }^{9}$ Con la denominación de sustentador principal del hogar, la EPF recoge para cada hogar aquel miembro del hogar de 16 años o más cuya aportación periódica (no ocasional) al presupuesto común se destina a atender los gastos del hogar en mayor grado que las aportaciones de cada uno de los restantes miembros. Aquí se han recogido, además de las personas registradas como tales, a sus cónyuges o parejas. Es decir, se utiliza para indicar en qué generación — padres o hijos— reside principalmente la dirección y responsabilidad del hogar. 


\section{Gráfico 3.}

Distribución de las generaciones de padres e hijos que conviven en función de quién ocupa la posición de sustentador principal.

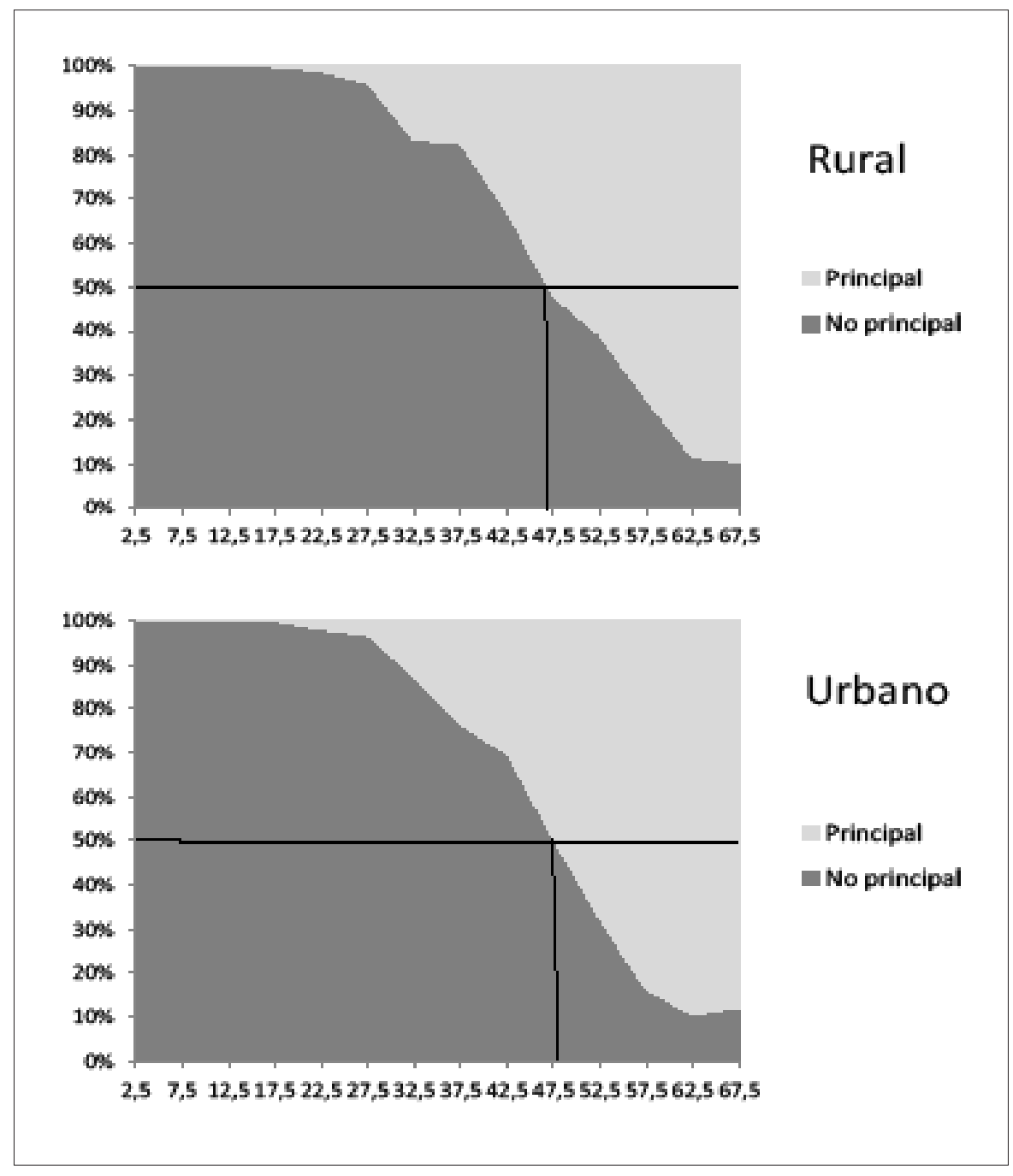

Fuente: Instituto Nacional de Estadística ([España] Encuesta de Presupuestos Familiares 2009). 
emancipación residencial de los jóvenes guarda relación con el alargamiento de su posición como hijos. Los hogares en los que existe convivencia generacional responden al modelo de hijos que no se han ido, antes que hogares de hijos que acogen a sus padres.

Los datos vienen a señalar asimismo que la convivencia está determinada por las redes de solidaridad y de apoyo entre generaciones. En el caso de España (Camarero et al. 2009) se ha observado que, por lo general, las redes de solidaridad familiar de las áreas rurales se conforman a través del ofrecimiento que hace la generación de los hijos de ayudas en transporte, en las tareas domésticas, así como en el cuidado de las personas mayores. De forma inversa, los mayores colaboran con sus hijos en el cuidado de los pequeños - nietos- o con ayudas económicas.

La persistencia de la convivencia generacional, en el caso de la España rural, así como la importancia creciente que tiene en su explicación -las redes de apoyo intergeneracionales - sugieren que nos encontramos ante un caso de mantenimiento de estructuras sociales aun cuando han tenido que modificar su funcionalidad. Para analizar esta cuestión en términos de resiliencia de los sistemas sociales vamos a indagar de forma más concreta en las estructuras y formas de convivencia sobre un grupo generacional concreto, al que en otros estudios (Camarero et al. 2009) hemos denominado la 'generación soporte'. La generación soporte es una generación - situada en torno a los 30-50 años- que por el efecto de los importantes desequilibrios demográficos de las áreas rurales resulta central tanto por su número como por su posición en la estructura social. Además, por su edad se encuentra en ese lugar que ha venido a denominarse "generación sándwich": aquellos que cuidan pequeños por estar en la edad de la crianza y a la vez cuidan mayores. $Y$ asimismo se enfrenta a desequilibrios de género muy relevantes. Los datos del Censo de 2001 ya apuntaban claramente, para los municipios rurales en sentido estricto, menores de 2.000 habitantes, una inusual proporción de varones que viven en hogares en la posición de hijo de la persona de referencia, fundamentalmente en las edades intermedias, como se aprecia en el gráfico 4.

Más recientemente, en el conjunto de las áreas rurales podemos observar que las dos terceras partes de las mujeres de la generación soporte viven con pareja y con hijos, es decir pertenecen a familias nucleares (tabla 4). La inclusión de las mujeres en áreas urbanas dentro de familias nucleares resulta bastante menor. En las áreas rurales también destaca el mayor número de mujeres rurales que viven con padres y con hijos a la vez en hogares trigeneracionales -joint family o familia patriarcal-, mientras por otra parte es casi insignificante el número de las que viven solas. Estos resultados señalan que las mujeres presentes en el medio rural lo están fundamentalmente a través de su rol de madres. Observemos que en total tres de cada cuatro mujeres de entre 30 y 50 años viven con hijos ${ }^{10}$. Los hombres rurales, presentan un perfil diferente: uno de cada seis vive con sus padres y no tiene pareja, reforzando la tendencia observada en 2001.

\footnotetext{
${ }^{10}$ Nucleares + Monomarentales + Trigeneracional $=76,1 \%$.
} 


\section{Gráfico 4.}

Porcentaje de varones en la posición de hijo de la persona de referencia* según edad y tamaño municipal en 2001

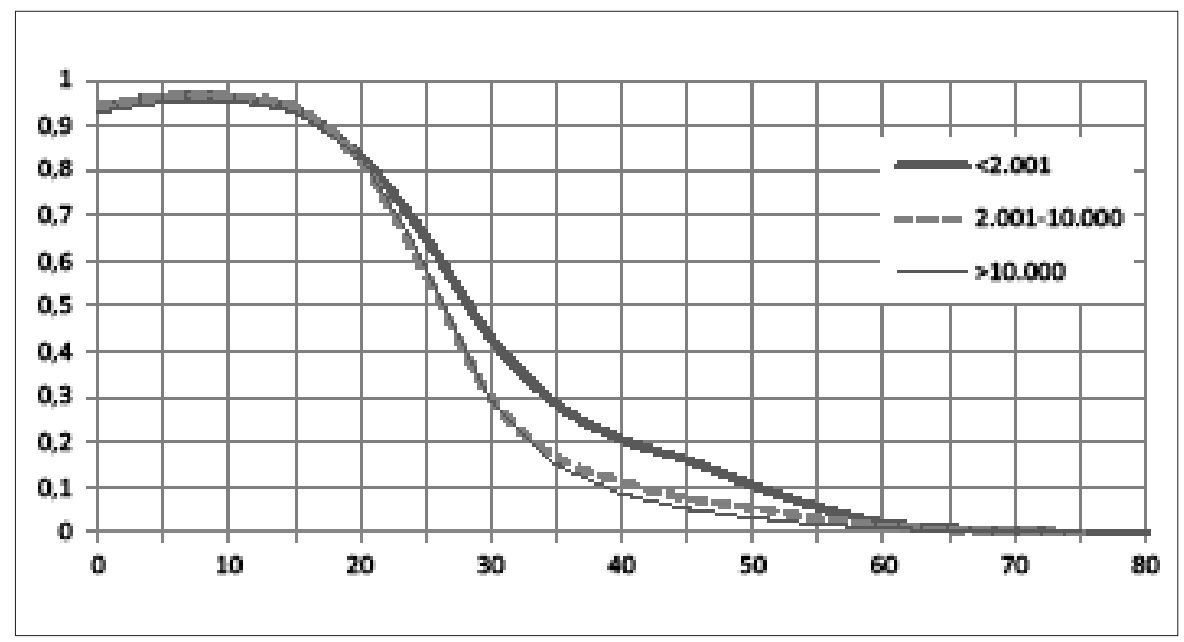

* En términos censales, la persona de referencia se elige, en primer lugar, por criterios familiares (convive con hijos o padres), seguidos por criterios de edad y estatus ocupacional, debiendo coincidir mayoritariamente con el sustentador principal o su pareja.

Fuente: Instituto Nacional de Estadística (Censo 2001).

Tabla 4.

Formas de convivencia de los miembros de la generación-soporte. (30-50 años) España.

\begin{tabular}{lcccc}
\hline & \multicolumn{2}{c}{ Rural } & \multicolumn{2}{c}{ Urbano } \\
\hline Solos & Hombres & Mujeres & Hombres & Mujeres \\
En pareja & $6,1 \%$ & $2,5 \%$ & $8,4 \%$ & $4,3 \%$ \\
En pareja con algún hijo & $11,6 \%$ & $10,7 \%$ & $14,5 \%$ & $13,1 \%$ \\
Padre/madre con algún hijo & $55,4 \%$ & $\mathbf{6 6 , 7} \%$ & $51,0 \%$ & $57,5 \%$ \\
Pareja con padres (sin hijos) & $0,5 \%$ & $2,9 \%$ & $0,4 \%$ & $\mathbf{5 , 1} \%$ \\
Solo con padres (sin hijos) & $0,7 \%$ & $1,0 \%$ & $0,6 \%$ & $0,7 \%$ \\
Familias patriarcales & $15,8 \%$ & $6,6 \%$ & $12,6 \%$ & $6,7 \%$ \\
Otros & $5,2 \%$ & $6,5 \%$ & $4,1 \%$ & $5,2 \%$ \\
TOTAL & $4,8 \%$ & $3,1 \%$ & $8,3 \%$ & $7,4 \%$ \\
\hline
\end{tabular}

Fuente: Instituto Nacional de Estadística (Encuesta de Presupuestos Familiares 2009). 
Estas diferencias de género no pueden deberse a las diferencias de edad de las parejas, que se sitúan como promedio en torno a los dos años, con tendencia a reducirse ${ }^{11}$, sino que hunden sus raíces en los desequilibrios demográficos señalados anteriormente: sobreenvejecimiento y masculinización. El peso de los mayores y los desequilibrios de género ponen en valor la estrategia de intercambio de apoyo entre generaciones entre los hijos varones respecto a sus padres.

Estos últimos datos muestran que hay un patrón de relaciones generacionales que se repite cuando no hay convivencia conyugal. Las mujeres conviven con descendientes mientras los hombres lo hacen con ascendientes. Esta asociación sugiere la preexistencia de un esquema de especialización de género en el ámbito de la reproducción doméstica; las mujeres en torno a la provisión de cuidados mientras que los hombres lo hacen en torno al funcionamiento y mantenimiento patrimonial.

\section{Líneas de debate. Hogares, estructuras, RESILIENCIA}

El análisis realizado ha mostrado la centralidad que tienen los procesos regulatorios de las relaciones intergeneracionales de convivencia. En concreto, pueden destacarse dos:

- Las poblaciones pueden acelerar o decelerar los procesos de emancipación de los jóvenes aumentando la convivencia intergeneracional.

- Las poblaciones pueden adelantar o retrasar la transición generacional a la dirección y organización de la vida doméstica.

Es decir, la relación de convivencia entre generaciones, además de la adscripción de género señalada anteriormente, constituyen los grados de libertad que tiene el sistema para adecuar el mantenimiento de las estructuras a la presión del entorno. En la modificación de estos parámetros — edad de emancipación y transición generacional- podemos observar la capacidad de resiliencia.

Así, en el caso de las áreas rurales de España se retrasa la independización y se retrasa la transición generacional. Es decir, las generaciones estiran al máximo el tiempo en el que pueden ser cuidadas. El efecto de irregularidad en la relación numérica entre grupos generacionales se resuelve aumentando la convivencia generacional.

El retraso en la transición entre generaciones, para maximizar el tiempo en el que uno es cuidado antes de convertirse en cuidador concuerda con el retraso efectivo, e incluso la ausencia, en la formación de parejas.

\footnotetext{
${ }^{11} \mathrm{El}$ efecto de la diferencia de edad de las parejas fue descartado al analizar la estructura de hogares, comparando los datos del Censo de 2001 y la Encuesta a Población Rural 2008, como base de la publicación de La población rural de España (Camarero et al. 2009).
} 
Los sistemas de familias rurales tradicionales se basaban en los tipos de familia patriarcal y troncal expuestos anteriormente (Ruggles 2010). En la medida en que en la actualidad las unidades domésticas rurales no tienen relación con la actividad familiar agraria se reduce la presencia de la "familia patriarcal" (familia extensa trigeneracional), dado que este tipo de organización pierde su funcionalidad. De la misma manera podría predecirse que la "familia troncal" perdería también su funcionalidad. Sin embargo, podemos considerar que el caso de los hijos solteros viviendo con sus padres es, en cierta medida, una "familia troncal" truncada. Es decir, es una rama a la que se le ha quitado el grupo nuclear de la segunda generación. Como podemos observar, este tipo de estructura no es únicamente rural sino que también tiene su importancia en áreas urbanas. Está claro que es un modelo resiliente pues mantiene la estructura de convivencia generacional desarrollando nuevas formas de solidaridad intergeneracional. No olvidemos que la estructura en la que se basaban los modelos tradicionales era la de generaciones que eran cada vez más numerosas. Ahora la relación entre generaciones resulta irregular y exige otro modelo de relación. Precisamente el sistema de interacción es el que se encarga de dotar de la nueva funcionalidad a las estructuras de convivencia generacional.

La relación de intercambio de ayudas entre generaciones en el interior de los hogares no está suficientemente estudiada. No obstante, parece lógico deducir que esta se basa en la solidaridad generacional. Los hijos estiran su tiempo de permanencia como tales recibiendo alojamiento y disfrutando de la comodidad del hogar paterno mientras participan en las tareas de transporte - fundamentales en las áreas rurales de pequeño tamaño y alta dispersión- así como en la realización de algunas tareas domésticas prácticas e incluso colaborando en tareas productivas.

En el medio rural de España este modelo resulta claramente visible. En cierta medida nos encontramos ante un "neomayorazgo", siendo el varón el que aspira a maximizar el tiempo en el que será cuidado mientras reside con los padres. Téngase en cuenta que en el gráfico anterior señalábamos que, sobre los 47 años, las generaciones empezaban a tener más peso como cabezas de familia en los hogares de convivencia intergeneracional, lo que supone que los padres en esos hogares tienen por regla general más de 67 años, y que en su gran mayoría son hogares que, progresivamente por las diferencias en esperanza de vida, se convierten en hogares de madres ancianas que viven con hijos solteros.

Otra cuestión de interés es la aproximación que se observa en los tipos de convivencia doméstica entre áreas rurales y urbanas. En este sentido es importante recordar, en primer lugar, la convergencia funcional de la familia rural y urbana. Antes la familia rural combinaba funciones productivas y reproductivas a la vez, mientras que la familia urbana era una estructura eminentemente reproductiva. Ahora, la familia rural, al perder su carácter productivo, se convierte únicamente en reproductiva también. Ahora son - exclusivamente - las condiciones de reproducción las que orientan las estructuras familiares. 
En segundo lugar, existen algunas condiciones demográficas comunes, como las relacionadas con el envejecimiento (aumento de la esperanza de vida y baja fecundidad). Estas condiciones hacen más probable un alargamiento del tiempo de convivencia intergeneracional, a la vez que modifican las estructuras de interacción que modelan los hogares y, especialmente, los procesos de emancipación residencial. A este respecto, Ruggles y Heggeness (2008) anotan el efecto que la menor competición entre herederos puede tener en la evolución de las estructuras domésticas, de modo que la menor presión entre miembros de la misma generación aumente las ventajas de la convivencia intergeneracional. También puede apuntarse el retraso en la transmisión patrimonial como consecuencia del alargamiento en la esperanza de vida.

Aunque la investigación ha expuesto la adaptación de las formas de hogar al entorno, especialmente al contexto demográfico, se abre a la vez la cuestión sobre la reformulación de las relaciones de emancipación y de separación doméstica entre generaciones. Así, puede apuntarse la progresiva pérdida de sentido de los hogares basados en la división sexual del trabajo, o de la capacidad de independencia económica con desvinculación absoluta de las formas domésticas; factores estos que permiten formas de convivencia más diversas. De este modo, la relativa convergencia de las formas de hogar observadas en los ámbitos rural y urbano aparece como pauta de una respuesta más general.

La siguiente cuestión parece clara: ¿Por qué se están separando también las áreas urbanas de los modelos modernizados de familia nuclear en los que la generación de los hijos se situaba como cabeza? Con idea de abrir nuevas líneas de trabajo, podríamos aventurar que el menor protagonismo que tiene la unidad familiar como unidad doméstica de supervivencia - en las áreas rurales como unidad productiva y, en las áreas urbanas, como unidad de convivencia basada en la división sexual del trabajo o male breadwinner model-, permite nuevas formas de hogar, que pueden quedar veladas por la hegemonía del modelo nuclear. En las áreas rurales, estas tendencias reducen la familia extensa pero reformulan el mayorazgo en clave reproductiva, mientras que en las áreas urbanas podrían vincularse a frenos en los procesos de emancipación económica, siendo el resultado la convergencia que observamos.

Por último, cabría preguntarse por la relación entre la resiliencia observada de las estructuras de hogar y las respuestas que las sociedades dan a la provisión del bienestar social, especialmente en el ámbito de la reproducción (Esping-Andersen 1990; 2008). $\mathrm{Si}$ el modelo de bienestar mediterráneo ya venía incorporando cambios importantes en el ámbito familiar, con la reducción de la fecundidad y las estrategias de capitalización patrimonial de las familias basadas en la propiedad residencial (Pérez-Díaz, Chuliá y Valiente 2000), el anunciado adelgazamiento del estado del bienestar conduce a una mayor familiarización de la vida cotidiana, pero, y ese es el misterio, con estructuras de hogar en las que los hijos se inhiben de tomar protagonismo. Probablemente los procesos de transición generacional temprana se encuentren asociados a la fortaleza de instituciones colectivas. 


\section{REFERENCIAS BibLIOGRÁfICAS}

Adger, N. 2000. "Social and ecological resilience: are they related?". Progress in Human Geography 24: $347-364$

Adger, N., M. Kelly, A. Winkels, L. Quangy and C. Locke. 2002. "Migration, Remittances, Livelihood Trajectories and Social Resilience". AMBIO 31: 358-366.

Agyeman, J. 2005. Sustainable Communities and the Challenge of Environmental Justice. Nueva York: Nueva York University Press.

Agyeman, J. y B. Evans. 2004. "Just Sustainability": The Emerging Discourse of Environmental Justice in Britain? The Geographical Journal 170: 155-146.

Beck, U. and E. Beck-Gernsheim. 2001. The Individualization. Thousand Oaks, CA: Sage.

Bennett, K. 2004. "A time for Change? Patriarchy, the Former Coaldfields and Family Farming". Sociologia Ruralis 44: 147-166.

Brown, D. 2008. "The Future of Rural America Through a Social-Demographic Lens". Pp. 229-248 en Frontiers in Resource and Rural Economics, edited by J. Wu, P. Barkley y B. Weber. Washington: Resources for the future.

Brown, D. y K. Schafft. 2011. Rural People \& Communities in the $21{ }^{\text {st }}$ Century. Resilience \& Transformation. Cambridge: Polity Press.

Camarero, L. 2005. Emprendedoras rurales. Valencia: Centro Tomás y Valiente.

Camarero, L., F. Cruz, F. González, J. del Pino, J. Oliva y R. Sampedro. 2009. The rural population in Spain. Barcelona: La Caixa.

(Del) Campo, S. 1982. La evolución de la familia española en el siglo XX. Madrid: Alianza.

(Del) Campo, S. y M. Navarro. 1985. Análisis sociológico de la familia española. Barcelona: Ariel.

Chayánov, A. V. [1925] 1986. The Theory of Peasant Economy. Chicago: The University of Wisconsin Press.

Díaz Méndez, C. 1997. Estrategias familiares y sociedad rural. Madrid: MAPA.

Esping-Andersen, G. 1990. The three worlds of welfare capitalism. Cambridge: Polity.

Esping-Andersen, G. 2008. Family formation and family dilemmas in contemporary Europe. Bilbao: Fundación BBVA.

Evans, G. 2008. "Transformation for 'Carbon Valley' to a 'Post-Carbon Society' in a Climate Change Hot Spot: the Coaldfields of the Hunter Valley, New South Sales, Australia". Ecology and Society 13:39.

Giddens, A. 1979. Central Problems in Social Theory. Action, Structure and Contradiction in Social Analysis. London: McMillan.

Gómez Reino. 1967. "La familia rural y urbana en España”. Pp: 3-46. Anales de Moral Social y Económica: La familia española, dirigida por Luis Sánchez Agesta. Madrid: CES Valle de los Caídos.

Gómez, C., J. González y R. Sancho. 1999. Identidad y profesión en la agricultura familiar. Madrid: Centro de Investigaciones Sociológicas. 
González, J. J., A. de Lucas y A. Ortí. 1985. Sociedad rural y juventud campesina. Madrid: MAPA.

Gunderson, L. y C. Holling. 2001. Panarchy: understanding transformations in human and natural systems. Washington: Island Press.

Hajnal, J. 1965. "European marriage in perspective". Pp.101-143 en Population in History, edited by D. V. Glass y D. E. C. Eversley. Chicago: Aldline.

Hermans, F. y L. Knippenberg. 2006. "A principle-based approach for the evaluation of sustainable development". Journal of Environmental Assessment Policy and Management 8: 299-320.

Holling, C. 2001. "Understanding the Complexity of Economic, Ecological, and Social Systems". Ecosystems 4: 309-405.

Iglesias, J. y L. Flaquer. 1993. "Familia y análisis sociológico: el caso de España". Revista Española de Investigaciones Sociológicas 61: 57-75.

Jurado, T. 2008. "Las nuevas familias españolas". Pp.59-88 en Tres décadas de cambio social en

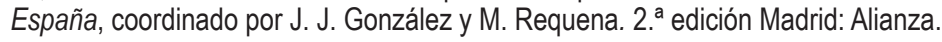

Keating, N., J. Swindley and S. Fletcher. 2011. "Aging in Rural Canada: A Retrospective and Review". Canadian Journal on Aging 30: 323-338.

Laslett, P. 1965. The World we Have Lost. Londres: Methuen.

Le Play, F. 1884. L'organisation de la familie selon le vrai modéle signalé par l'histoire de toutes les races et de tous les temps. Tours: A. Mame.

Luhmann, N. 1995. Social Systems. Standford: Standford University Press.

Mannhein, K. 1952. "The problem of generations". Pp. 276-320 in Essays on the Sociology of Knowledge, edited by K. Manhein. London: Routledge and Kegan Paul.

Marsden, T. 2008. Sustainable Communities. Oxford: Elsevier.

Marsden, T. and F. Hines. 2008. "Unpacking the new quest for community: some conceptual parameters". Pp. 21-44 in Sustainable Communities, edited by T. Marsden. Oxford: Elsevier.

McGinnis, M. 1999. Bioregionalism. London: Routledge.

McManus, P., J. Walmsley, N. Argent, S. Baum, L. Bourke, J. Martin, B. Pritchardy and T. Sorensen. 2012. "Rural Community and Rural Resilience: What is important to farmers in keeping their country towns alive?". Journal of Rural Studies 28: 20-29.

(De) Pablo Masa, A. 1970. "La familia española en cambio". Pp.345-406 en FOESSA Informe de la situación social de España. Madrid: Euramérica.

Parsons, T. 1949. "The social structure of the family". Pp.173-201 in The family: its function and destiny, edited by R. N. Nashen. Nueva York: Harper.

Pelling, M. and D. Manuel-Navarrete. 2011. "From Resilience to Transformation: The Adaptative Cycle in Two Mexican Urban Centers". Ecology and Society 16:11. Consulta (http://www.ecologyandsociety.org/vol16/iss2/art11/).

Pérez-Díaz, V., E. Chuliá y C. Valiente. 2000. La familia española en el año 2000. Madrid: Fundación Argentaria y Visor. 
Pilcher, J. 1995. Age and Generation in Modern Britain. Oxford: Oxford University Press.

Rawls, J. 1999. A Theory of Justice. Oxford: Oxford University Press.

Ruggles, S. 2009. "Reconsidering the Northwest European Family System: Living Arrangemente of the Aged in Comparative Historical Perspective". Population Development Review 35: 249-273.

Ruggles, S. 2010. "Stem Families and Joint Families in Comparative Historical Perspective". Population and Development Review 36: 563-577.

Ruggles, S. and M. Heggeness. 2008. "Intergeneracional Coresidence in Developing Countries". Population and Development Review 34: 253-281.

Sancho Hazak, R. 1972. "La sociedad rural hoy". Pp. 219-317 en La España de los años 70, vol. 1, La sociedad, editado por M. Fraga y S. del Campo. Madrid: Moneda y Crédito.

Scott, K., J. Parky and C. Cocklin. 2000. "From 'sustainable rural communities' to 'social sustainability': giving voice to diversity in Mangakahia Valley, New Zealand". Journal of Rural Studies 16: 433-446.

Sgro, C., A. Lowe y A. Hoffmann. 2011. "Building evolutionary resilience for conserving biodiversity under climate change". Evolutionary Applications 4: 326-337.

Solsona, M. y R. Treviño. 1990. Estructuras familiares en España. Madrid: Instituto de la Mujer.

Stern, N. 2007. The Economics of Climate Change. Cambridge: Cambridge University Press.

Trabalzi, F. y M. de Rosa. 2012. "Market and State-supported Sustainability: A Tale of Two Rural communities in lowa and Italy". Sociologia Ruralis 52: 115-133.

Van de Kaa, D. J. 1987. "Europe's Second Demographic Transition”. Population Bulletin 42.

Verdon, M. 1979. "The stem family: toward a general theory". The Journal of Interdisciplinary History 10: $87-105$.

Walker, B. y D. Salt. 2006. Resilience Thinking: Sustaining Ecosystems and People en a Changing World. Washington: Island Press.

WCED. 1987. Report of the World Commission on Environment and Development: Our Common Future. Oxford: Oxford University Press.

Williams, J. 2001. "Achieving Local Sustainablity in Rural Comunities". Pp. 235-252 in Planning for a Sustainable Future, edited by A. Layard, S. Davoudi and S. Batty . London: Spon Press.

Luis Camarero Rioja es Catedrático en el Departamento de Teoría, Metodología y Cambio Social de la UNED. Desarrolla su labor docente en el ámbito de la estadística y las técnicas de investigación. Como investigador ha dirigido y participado en distintos proyectos y contratos de investigación sobre procesos sociodemográficos y territoriales, temas en los que ha publicado más de medio centenar de textos. Ha sido Visiting Scholar en la City and Regional Planning School (Wales University) y Director del Departamento de Sociología I.

Julio A. del Pino Artacho es Licenciado y Doctor en Ciencias Políticas y Sociología. Especialista en Investigación Social Aplicada y Análisis de Datos. Profesor del Departamento de Sociología I de la UNED. Ha combinado su docencia en Teoría Sociológica con la investigación acerca 
de los procesos demográficos y territoriales en la era de la movilidad. Ha participado como investigador en una decena de proyectos (competitivos o a través de contratos), dando lugar a una treintena de publicaciones.

RECIBIDO: $27 / 12 / 2012$

ACEPTADO: $30 / 07 / 2013$

Publicado on-line: $24 / 04 / 2014$ 